\title{
Viscosity of a Newtonian fluid calculated from the deformation of droplets covered with a surfactant under a linear shear flow
}

Journal Article

Author(s):

Megias-Alguacil, David; Windhab, Erich J.

Publication date:

2006-12

Permanent link:

https://doi.org/10.3929/ethz-b-000035668

Rights / license:

In Copyright - Non-Commercial Use Permitted

Originally published in:

Rheologica Acta 46(2), https://doi.org/10.1007/s00397-006-0122-2 


\title{
Viscosity of a Newtonian fluid calculated from the deformation of droplets covered with a surfactant under a linear shear flow
}

\author{
David Megias-Alguacil • Erich J. Windhab
}

Received: 23 November 2005 / Accepted: 29 June 2006 / Published online: 31 August 2006

(C) Springer-Verlag 2006

\begin{abstract}
The viscosity of small fluid droplets covered with a surfactant is determined using drop deformation techniques. This method, proposed by Hu and Lips, is here extended to the case of the presence of a surface-active adsorpted at the liquid-liquid interface, to consider more general scenarios. In these experiments, a droplet is sheared by another immiscible fluid of known viscosity, both Newtonian liquids. From the steady-state deformation and retraction mechanisms, the droplet viscosity is calculated using an equation derived from the theories of Taylor and Rallison. Although these theories were expressed for surfactant-free interfaces, they can be applied when a surfactant is present in the system if the sheared droplet reaches reliable steady-state deformations and the surfactant attains its equilibrium adsorption concentration. These determinations are compared to bulk viscosities measured in a rheometer for systems with different viscosity ratios and surfactant concentrations. Very good agreement between both determinations is found for drops more viscous than the continuous phase.
\end{abstract}

Keywords Viscosity · Drop deformation - Relaxation . Surfactant $\cdot$ Shear flow

D. Megias-Alguacil $(\bowtie) \cdot$ E. J. Windhab

Laboratory of Food Process Engineering,

Institute of Food Science and Nutrition,

Swiss Federal Institute of Technology ETH-Zürich,

8092 Zürich, Switzerland

e-mail: david.megias@ilw.agrl.ethz.ch

\section{Introduction}

The drop deformation technique has been revealed to be a useful method in determining interfacial properties of liquid-liquid systems because the shape of the interface between the two media can be optically tracked along the experimental time and further digitally analysed (MegiasAlguacil et al. 2004a). This technique consists of shearing a single droplet immersed in a matrix fluid, in which the drop is immiscible. The flow is applied by the movement of two belts in opposite directions, thus creating a simple linear flow. Under the action of the flow, the droplet deforms and also orients until it reaches a steady state of deformation and orientation, which can be maintained indefinitely along the experimental time if the flow conditions remain unaltered.

According to the previous work of $\mathrm{Hu}$ and Lips (2001), this experimental technique provides a useful method for measuring the viscosity of very small amounts of a fluid (order of some microlitres). This is of special practical interest when the available amount of the problem liquid is limited (high cost, difficult production or synthesis, etc.) and it is not able to fill the measuring cell volume of a conventional rheometer.

The deformation of a sheared droplet, as well as its relaxation to the spherical shape after cessation of the flow, is governed by the type of flow and the physical-chemical properties of the system, mainly the viscosity ratio, $\lambda=\eta_{\mathrm{d}} / \eta_{\mathrm{m}}$, for the disperse $\left(\eta_{\mathrm{d}}\right)$ to the continuous fluid phase $\left(\eta_{\mathrm{m}}\right)$, and the interfacial tension of the liquid-liquid interface, $\sigma$. The deformation of such a drop occurs when the stress exerted on the drop by the flow is high enough to overcome the Laplace pressure. The ratio of these opposing stresses is 
known as the capillary number, $\mathrm{Ca}$, defined for laminar flows by:

$C a=\frac{\eta_{\mathrm{m}} \dot{\gamma} R}{\sigma}$

where $\eta_{\mathrm{m}}$ is the viscosity of the continuous fluid phase, $\dot{\gamma}$ is the applied shear-rate, $R$ is the radius of the undeformed droplet, and $\sigma$ is the interfacial tension of the liquid-liquid interface.

To avoid the previous knowledge, or measurement of the interfacial tension present in Eq. 1, which is not always known or easily accessible, it is necessary to consider both droplet deformation and relaxation mechanisms. Then, coupling the equations of the models that describe these dynamical effects, $\sigma$ mathematically vanishes in the final expression (Hu and Lips 2001).

The first mechanism was firstly described by Taylor (1934), whose model for the steady-state deformation linearly depends on $\mathrm{Ca}$, reading as follows:

$\mathrm{D}=\frac{19 \lambda+16}{16 \lambda+16} \mathrm{Ca}$

where $D=(L-B) /(L+B)$ is the deformation parameter, $L$ and $B$ being the half-lengths of the sheared droplet in the flow direction and gradient direction, respectively.

Once the droplet has reached the steady-state by the action of the applied shear flow, if the flow is then stopped, the droplet retracts under the action of the interfacial forces to the initial spherical shape. This mechanism is described by an exponential decay. Limiting the initial deformation to be small, the absolute value of the characteristic time of such exponential decay is given, according to the model of Rallison (Rallison 1984; Guido and Villone 1999), by:

$\alpha=\frac{(19 \lambda+16)(2 \lambda+3)}{40(\lambda+1)} \frac{\eta_{\mathrm{m}} R}{\sigma}$

Combining Eqs. 1-3, and solving for $\lambda, \mathrm{Hu}$ and Lips (2001) derived the following expression for the viscosity of the droplet submerged in a fluid of known viscosity and submitted to a simple shear flow:

$\eta_{\mathrm{d}}=\left(\frac{5}{4} \frac{\alpha \dot{\gamma}}{D_{\mathrm{S}}}+\frac{3}{2}\right) \eta_{\mathrm{m}}$

where $D_{\mathrm{s}}$ is the steady value of the droplet deformation. As mentioned previously, this equation does not depend explicitly on the interfacial tension of the liquid-liquid interface, as well as the initial drop radius. Nevertheless, in practical terms, the size of the droplet is limited by the dimensions of the gap between the belts.
Also, simple inspection of Eq. 4 provides the range of shear rates that can be considered in the calculation of $\eta_{\mathrm{d}}$. Indeed, because the characteristic relaxation time, $\alpha$, does not depend on $\dot{\gamma}$, according to Eq. 3, the ratio $\dot{\gamma} / D_{\mathrm{s}}$ must be a constant for a particular system. Thus, only values of deformation in the linear region of the plot $D_{\mathrm{s}}$ vs $\dot{\gamma}$ can be considered. Further, the slope of such a linear region, $S_{\text {Taylor, }}$, allows us to re-write Eq. 4 in terms of just constant values, which gives a more intuitive view for the expression of the viscosity of a Newtonian fluid:

$\eta_{\mathrm{d}}=\left(\frac{5}{4} \frac{\alpha}{S_{\text {Taylor }}}+\frac{3}{2}\right) \eta_{\mathrm{m}}$

It is well known that the deformation parameter follows such a linear behaviour with respect to the capillary number, $\mathrm{Ca}$ - which, in fact, is a dimensionless expression for the shear rate - up to a certain value. From this value, $D$ diverges (when $\lambda<1$ ), indicating that the drop break-up is possible or tends to a finite asymptotic limiting value (when $\lambda>1$ ) (Rumscheidt and Mason 1961). In certain cases, this limiting value is found to be below the usually accepted range of validity of the theory of Taylor, Eq. 2, namely, $D<0.20$ (Wolf et al. 2001). For the purposes of the present investigation, the restriction of the linearity of $D$ must be carefully observed. In practical terms, this implies that several experiments at different shear rates should be carried out to determine the appropriate range of valid $\dot{\gamma}$, despite the fact that just one single experiment should be considered to be enough on the view of Eq. 5 .

It is interesting to point out that from a two-dimensional characteristic of the system, the liquid-liquid interface, it is possible to obtain information of a three-dimensional property, the bulk viscosity.

We already tested in a preliminary work the expression by $\mathrm{Hu}$ and Lips, Eq. 4, finding a good agreement with respect to the measurements carried out in a conventional rheometer (Megias-Alguacil et al. 2004b). It is our purpose now to extend its use to systems that may contain a surfactant covering the liquid-liquid interface, even when the previously mentioned models were not firstly developed for this circumstance. Their application when a surfaceactive substance is present in the system is fully justified under the observance of the following requisites: (1) the adsorption of the surfactant at the liquid-liquid interface has reached the equilibrium and (2) steady states in the linear regions of deformation are accurately developed.

When these conditions are satisfied, the sheared interface does not vary in time after reaching the stationary state, thus making possible the application of the theories. Even more, under the equilibrium conditions 1 and 2, a system containing a surfactant is dynamically equivalent to another "surfactant-free" one with a reduced interfacial tension, for which the models can be used. This procedure is supported 
by its successful application when calculating the interfacial tension of similar systems containing surfactants by means of drop deformation techniques (Megias-Alguacil et al. 2006).

\section{Materials and methods}

The experimental setup consists of a parallel band apparatus, built in our laboratory. The apparatus has two metallic belts that run around several rolls to allow the adjustment of the gap between the two belts and also to keep them straight. The belts are spring-loaded to avoid any bending effect. Their dimensions are $170.00 \pm 0.05 \mathrm{~mm}$ in length and $33.00 \pm 0.05 \mathrm{~mm}$ in width. Their motion is computercontrolled independently through two motors, as described elsewhere (Birkhofer et al. 2005). The time sequence of the droplets' behaviour is tracked with two CCD digital cameras (Sony DFW-V500, Tokyo, Japan), one placed in a vertical position with respect to the device and the other aligned within the flow direction.

The flow profile developed in the parallel bands apparatus is linear and the shear rate was simply obtained as the ratio between the relative speed at which the belts move and the distance between them. In our experiments, the droplets were deformed under the action of several shear rates in the range $\dot{\gamma} \leq 30 \mathrm{~s}^{-1}$ selected by controlling both magnitudes. The radii of the undeformed droplets were adjusted with respect to the belts gap to consider negligible any edge effect.

In the present investigations, binary systems dropletcontinuous phases were used. As continuous phase, we have employed two different polymer solutions, both transparent: (a) a solution of $15 \%$ by weight of polyvinylpyrrolidone (Fluka, Deisenhofen, Germany) in water, further named as "PVP", with a density of $\rho=1.03 \mathrm{~g} / \mathrm{cm}^{3}$. Two amounts $(0.1$ and $1 \%$ by weight) of a non-ionic water-soluble surfactant "Tween 20" (Fluka) were later added to the continuous phase. This surfactant is a polyoxyethylene sorbitan monolaurate. (b) Another Newtonian solution of polyethylene glycol-water-ethanol, further named as "PWE", with a density of $\rho=0.97 \mathrm{~g} / \mathrm{cm}^{3}$ was employed. Three amounts $(0.125,0.250$ and $0.500 \% \mathrm{w} / \mathrm{w})$ of a water-soluble surfactant "SOP/03" (Kolb, Hedingen, Switzerland) were added to this continuous phase. This surface-active agent is composed of a blend of alkoxylated alcohol and ester.

The dispersed drops in both continuous phases were made of different silicon oils (Wacker, Munich, Germany); these oils are Newtonian and they are available with different viscosities, maintaining constant other properties like interfacial tension and density. To avoid vertical displacements of the droplets, which could lead to a loss of optical resolution, adequate amounts of titanium oxide were added to the oils to fit their densities to those of the continuous phases and to make the droplets opaque, thus providing sufficient optical contrast for analysis.

The bulk viscosities of the silicon oils, as well as the fluid matrixes, were determined with a Rheometrics dynamic stress rheometer. The measuring system was a concentric cylinders device whose inner and outer radii were $29.51 \pm$ 0.02 and $31.98 \pm 0.02 \mathrm{~mm}$, respectively. Such determinations showed a Newtonian behaviour of all the substances in a wider range of shear rates than those available in our parallel bands apparatus. The values for the continuous fluid phases were found to be $\eta_{\mathrm{m}}=325 \pm 5 \mathrm{mPa} \cdot \mathrm{s}$ for the PVP solution and $\eta_{\mathrm{m}}=290 \pm 5 \mathrm{mPa} \cdot \mathrm{s}$ for the PWE solution.

The addition of titanium oxide to the oils slightly changed their original viscosities, being different in both sets of systems, as displayed in Table 1. The presence of the surfactants in the corresponding continuous phases did not perceptibly change the viscosities of the continuous phases because of the small added amounts. All experiments were carried out at $25^{\circ} \mathrm{C}$.

\section{Results and discussion}

The flow used in this paper belongs to the plane flow type, and the droplet deforms in all three directions. Indeed, direct observations along the flow direction allowed the determination of the ratio $W / R$, where $W$ is the drop halflength along the vorticity direction. Even though $W$ is not involved in any calculation of the drop viscosity (recall that just $L$ and $B$ are needed for determining the deformation parameter, $D$ ), let us here briefly discuss it. Figure 1 displays the steady droplet deformation along the vorticity direction for the cases AK 100 and AK 1,000, in pure PVP solution and when $c=0.1 \% \mathrm{w} / \mathrm{w}$ of Tween 20 . As shown, the ratio $W / R<1$ indicates that the drop flatters along this third direction, then becomes an oblate ellipsoid. As expected, this deformation increases (smaller $W / R$ ratios)

Table 1 Viscosities and viscosity ratios, $\lambda=\eta_{\mathrm{d}} / \eta_{\mathrm{m}}$, of the different silicon oils (droplet) with respect to the two different continuous phases

\begin{tabular}{|c|c|c|c|c|}
\hline \multirow[t]{2}{*}{ Silicon oil } & \multicolumn{2}{|c|}{ PVP solution } & \multicolumn{2}{|c|}{ PWE solution } \\
\hline & $\eta(\mathrm{mPa} \cdot \mathrm{s})$ & $\lambda$ & $\eta(\mathrm{mPa} \cdot \mathrm{s})$ & $\lambda$ \\
\hline AK 50 & $50 \pm 1$ & $(0.15)$ & $48 \pm 1$ & $(0.17)$ \\
\hline AK 100 & $109 \pm 1$ & $(0.3)$ & $96 \pm 1$ & $(0.3)$ \\
\hline AK 1,000 & $1,084 \pm 5$ & (3.3) & $970 \pm 5$ & (3.4) \\
\hline AK 2,000 & $2,060 \pm 8$ & (6.3) & $1,940 \pm 7$ & $(6.7)$ \\
\hline AK 5,000 & $5,190 \pm 50$ & (16) & $4,850 \pm 30$ & (17) \\
\hline AK 10,000 & $9,740 \pm 90$ & $(30)$ & $9,700 \pm 70$ & (33) \\
\hline
\end{tabular}

$\eta_{\mathrm{PVP}}=(325 \pm 5) \mathrm{mPa} \cdot \mathrm{s}$

$\eta_{\mathrm{PWE}}=(290 \pm 5) \mathrm{mPa} \cdot \mathrm{s}$ 


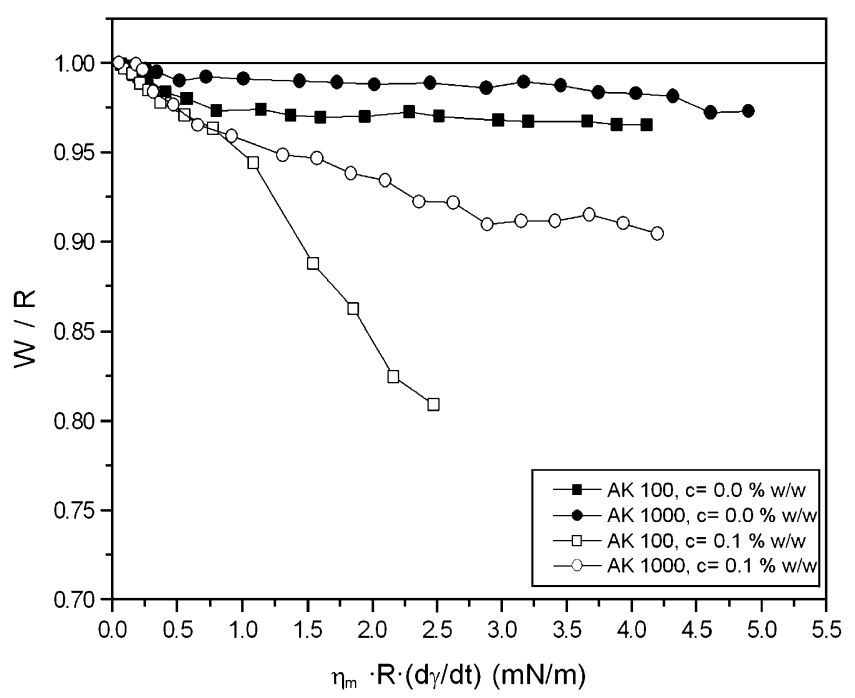

Fig. 1 Deformation along the third direction, $W / R$, as a function of the applied shear rate (times the fluid matrix viscosity and the drop unsheared radius) for AK 100 and AK 1,000 in PVP solution, without surfactant and with a concentration of $c=0.1 \% \mathrm{w} / \mathrm{w}$ of Tween 20

with increasing $\dot{\gamma}$, as well as increasing the amount of surfactant (lowering of interfacial tension). In the same manner, the ratio $W / R$ gets smaller for the less viscous drops.

The observation of the drop deformation from a vertical view enables the determination of both half-lengths of the sheared droplet in the flow direction, $L$, and gradient direction, $B$. From these magnitudes, the droplet deformation parameter, $D$, was measured as a function of the experimental time at each shear-rate value for the corresponding system.

Figure 2 shows some examples of such experiments at various shear-rates, $\dot{\gamma}$, for the systems $\operatorname{AK} 1,000(\lambda=3.3)$ in PVP when the surfactant concentration is $c=0.1 \% \mathrm{w} / \mathrm{w}$ (Fig. 2a) and AK $100(\lambda=0.3)$ in pure PWE (Fig. 2b). As observed in all the cases, the initially spherical droplet $(D=0)$ deforms by the action of the flow until it reaches a steady deformation, $D_{\mathrm{s}}$, which lasts while the flow conditions remains the same. As expected, the steady value of the deformation parameter increases with increasing the shear-rate. After cessation of the flow, the droplet retracts, recovering the spherical shape. Notice that, in all the cases, a reliable steady state of deformation is fully reached, as indicated by the presence of a plateau of $D$. When a surfactant covers the interface (Fig. 2a), the steady deformation of the droplets is also attained, which, in turn, demonstrates an equilibrium adsorption of such substance at the drop surface. This steady-state is also an indication of the equilibrium between the competing Marangoni stresses and surface dilation effects, which influence the dynamics of the interface in the presence of surfactants (Pawar and Stebe 1996; Eggleton et al. 1999). a

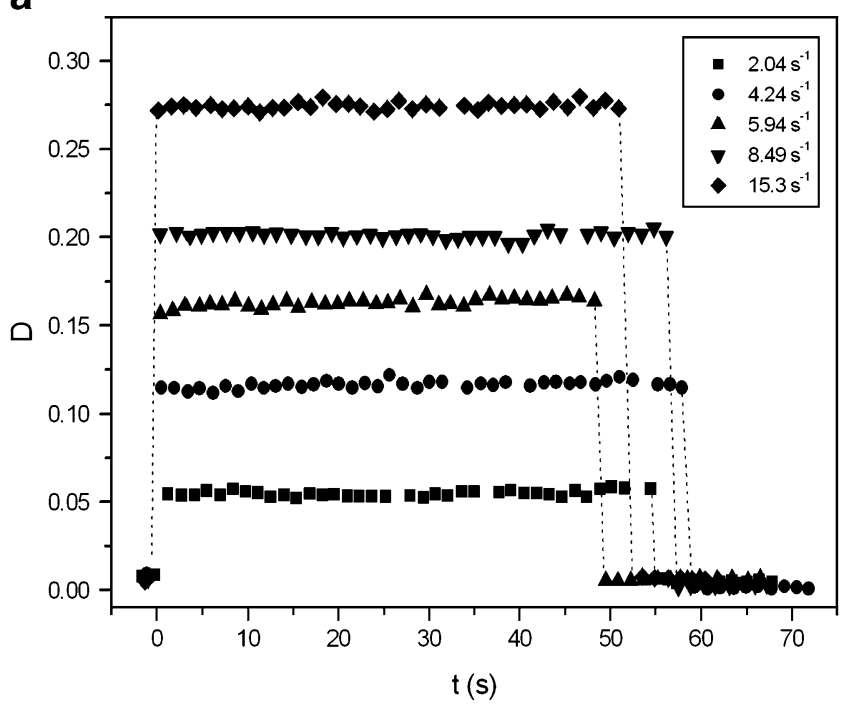

b

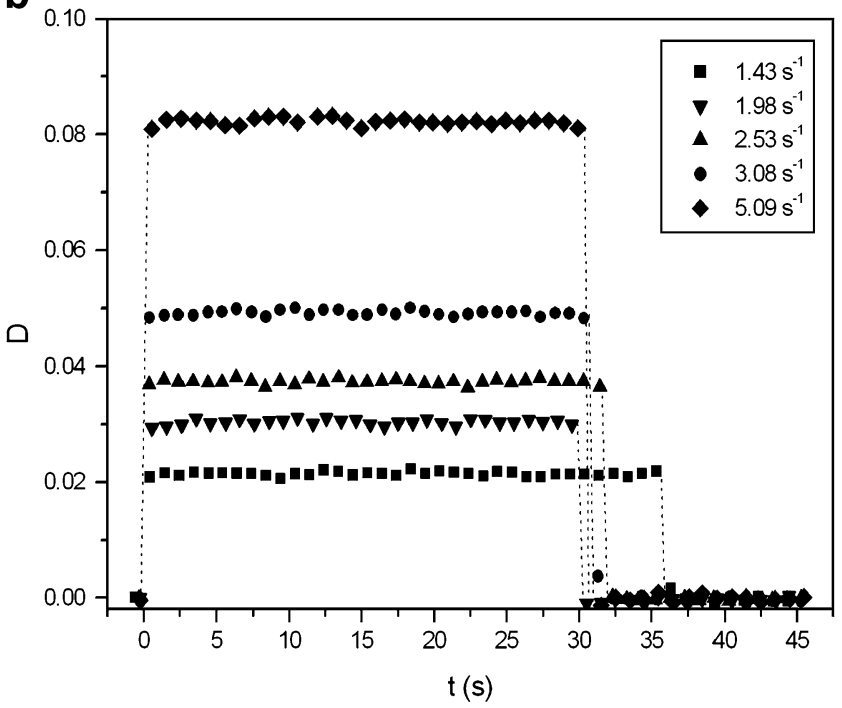

Fig. 2 Time evolution of the deformation parameter, $D$, at the indicated shear rates, $\dot{\gamma}$. a AK 1,000 in PVP solution with $c=0.1 \% \mathrm{w} / \mathrm{w}$. b AK 100 in pure PWE solution

As mentioned above, the retraction mechanism of the sheared drop to the spherical shape is governed by an exponential decay with a characteristic time, $\alpha$, that can be calculated from a semi-log plotting of $D$ vs $t>t_{\text {off-flow, }}$ as shown in Fig. 3, for the cases AK 10,000 $(\lambda=30)$ in PVP when the surfactant concentration is $c=0.1 \% \mathrm{w} / \mathrm{w}$ (Fig. 3a) and AK 5,000 $(\lambda=17)$ in PWE when the surfactant concentration is $c=0.5 \% \mathrm{w} / \mathrm{w}$ (Fig. $3 b$ ). Due to the independence of the relaxation process with respect to the shear rate, Eq. 3, the deformations decrease from the corresponding steady-state values in parallel lines (Fig. 3) in the semi-log plot. Changes in the surfactant concentration that may affect the relaxation rate are not expected due to the short times involved in the recovery processes.

The effect of the surfactant concentration and drop viscosity on the dynamical behaviour of the droplets can be 


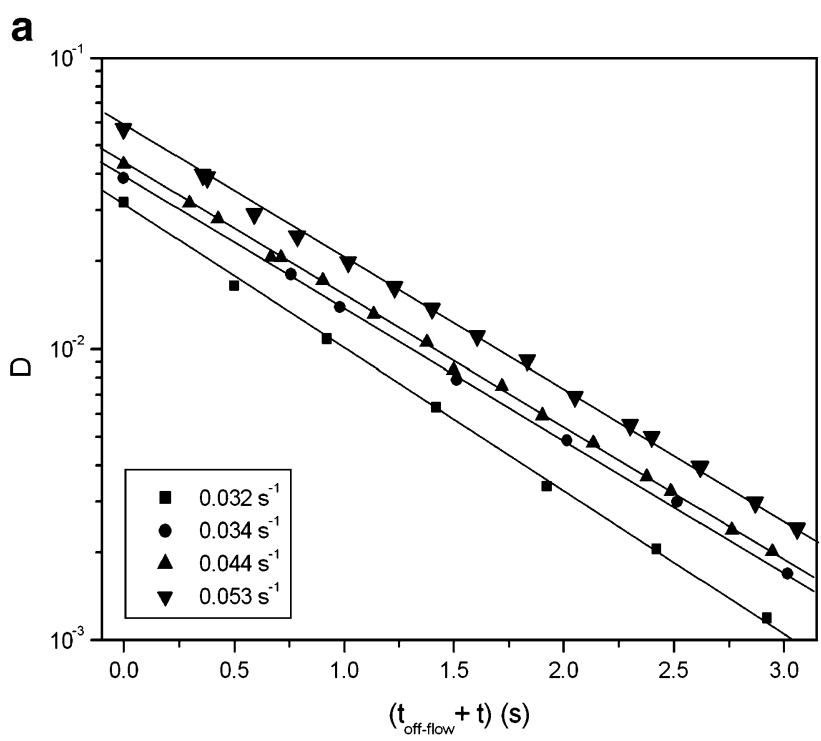

b

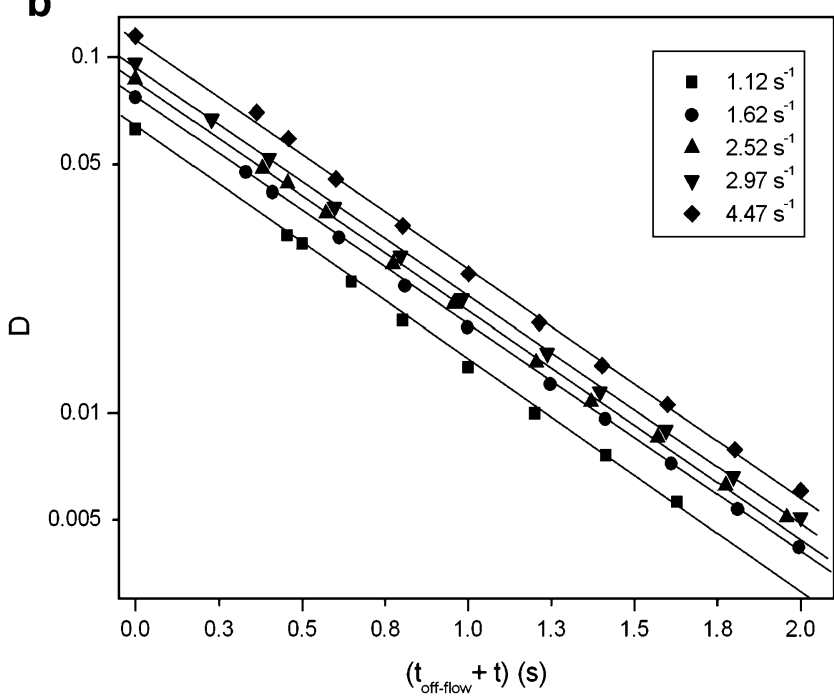

Fig. 3 Semi-log plotting of $D$ after the removal of the shear flow. The time has been shifted to the point of flow cessation, $t_{\text {off-flow. The }}$ legend indicates the shear-rates applied prior to flow stop. The solid lines represent the exponential decay followed by each droplet. a AK 10,000 in PVP solution, $c=1.0 \% \mathrm{w} / \mathrm{w}$. b AK 5,000 in PWE solution, $c=0.5 \% \mathrm{w} / \mathrm{w}$

observed from Fig. 4, where we have plotted the steady state of deformation, $D_{\mathrm{s}}$, as well as the relaxation characteristic time, $\alpha$, as a function of the product $\left(\eta_{\mathrm{m}} R \dot{\gamma}\right)$ to avoid the influence of the drop initial radius, $R$, in the following discussion.

As observed in Fig. 4a for the case AK 2,000 $(\lambda=6.3)$ and the three different concentrations of Tween 20, the surfactant induces higher deformations on the drop at the same conditions than for the surfactant-free interface, also increasing the slopes (dashed lines in Fig. 4a) of the linear regions of the steady deformation, $S_{\text {Taylor }}$, corresponding to each surfactant concentration. These facts indicate the effectiveness of the adsorption of the surface active to the a

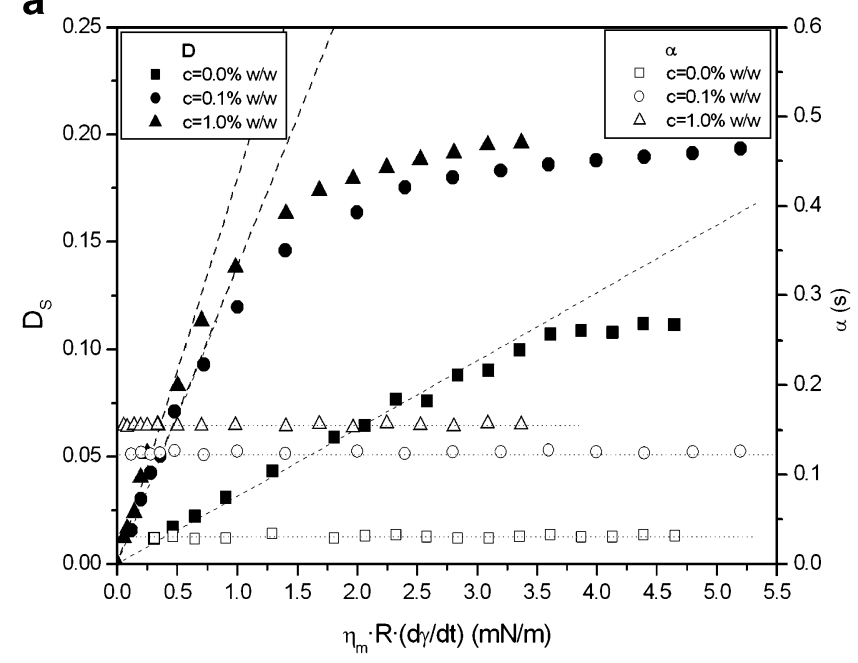

b

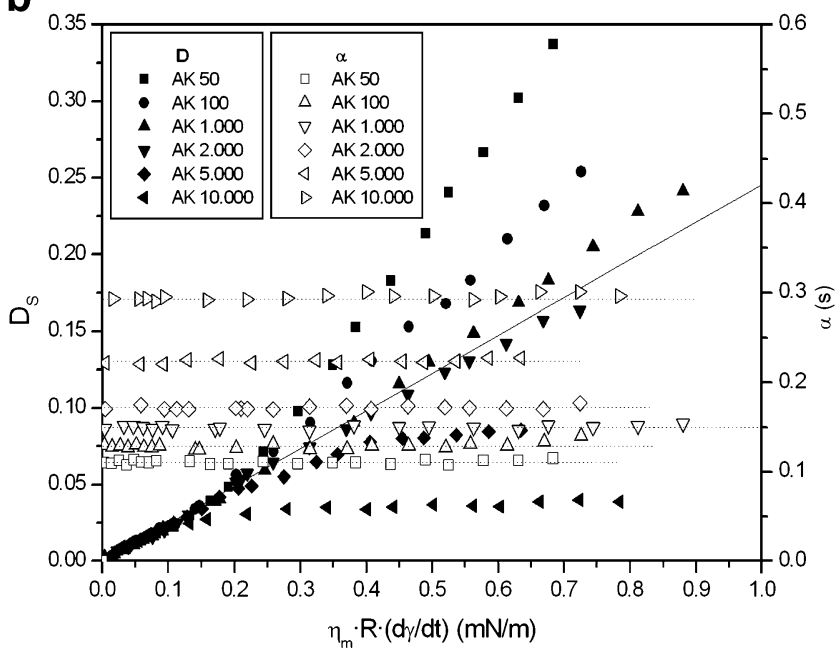

Fig. 4 Steady deformation parameter, $D_{\mathrm{s}}$, and characteristic relaxation time, $\alpha$, as a function of the applied shear rate (times the fluid matrix viscosity and the drop unsheared radius). Dashed lines correspond to linear fits according to the model of Taylor. Dotted lines indicate the mean values of $\alpha$. a AK 2,000 in PVP solution for the different concentrations of Tween 20. b AKs in PWE solution at $c=0.250 \% \mathrm{w} / \mathrm{w}$

liquid-liquid interface, thus lowering the interfacial tension, $\sigma$, when increasing the amount of surfactant in the system.

In the same manner, the reduction of the interfacial tension is followed by a slower recovery of the sheared drop to the spherical shape, or in other terms, the increase of $\alpha$ according to Eq. 3, as shown in Fig. 4a. The values of the relaxation times lay around their mean value (dotted lines) for the whole range of shear-rates applied, in accordance with the previous results shown in Fig. 3.

The steady deformation parameter, $D_{\mathrm{s}}$, increases with increasing the applied shear rate. Initially, this increment follows the linear behaviour predicted by Eq. 2. At a fixed surfactant concentration, like that shown in Fig. 4b, the linear dependencies of $D_{\mathrm{s}}$ vs Ca, Eq. 1, almost overlap for all the oils (just one line has been plotted in Fig. $4 \mathrm{~b}$ for the 
sake of clarity) because $D_{\mathrm{s}}$ in this region slightly increases with respect to the viscosity ratio, $\lambda$, in agreement with the model of Taylor. This overlapping also indicates that the interfacial tension, $\sigma$, is the same for all the silicon oils at a certain surfactant concentration.

Beyond the linear region, it is found that for the droplets less viscous than the fluid matrix $(\lambda<1), D_{\mathrm{s}}$ diverges (concave behaviour with respect to the capillary number, $\mathrm{Ca}$ ), indicating an eventual break-up of the droplet at sufficient flow strength (Grace 1982). Observe also in Fig. 1 the pronounced deformation along the third axis for the systems with lower drop viscosity and higher surfactant concentration. On the other hand, for drops more viscous than the fluid matrix $(\lambda>1), D_{\mathrm{s}}$ tends towards limiting values after the linear region (see Fig. 4a,b). These limiting values of deformation increase when the viscosity ratio, $\lambda$, decreases and/or the surfactant concentration increases (in other words, when the interfacial tension decreases). Notice than in such cases, the extents of the linear dependences are below 0.20 . In practical terms, only values of steady deformation, $D_{\mathrm{s}}$, inside the corresponding linear regions will be considered in further calculations, according to our working hypothesis, above explained.

The impact of the drop viscosity, $\eta_{\mathrm{d}}$, on the relaxation time, $\alpha$, is shown in Fig. 4b. As displayed, $\alpha$ increases with increasing the drop viscosity, in agreement with Eq. 3, which predicts a first-order dependence of $\alpha$ with $\eta_{\mathrm{d}}$ (recall the viscosity ratio, $\lambda=\eta_{\mathrm{d}}\left(\eta_{\mathrm{m}}\right)$.

Once both magnitudes, $D_{\mathrm{s}}$ and $\alpha$, are experimentally determined at the corresponding conditions of viscosity ratio, $\lambda$, surfactant concentration, $c$, and shear rate, $\dot{\gamma}$, the viscosity of the droplet fluid is calculated by means of Eq. 4 . Averaged values of such calculated viscosities at different shear-rates are plotted for each silicon oil immersed in the PVP solution (Fig. 5a) and in the PWE solution (Fig. 5b), and then compared with the data of bulk viscosity for the same liquids measured in the conventional rheometer.

Figure 5 also shows the results when the surfactant is present in the corresponding systems. The agreement between the drop deformation technique and the measured viscosities in the rheometer is qualitative and quantitatively very good, with values almost coincident with those of the non-surfactant case. This fact probes the independency of Eq. 4 respect to the interfacial tension.

We observe for our systems that the agreement between both methods is only found for drop viscosities higher than the fluid matrix viscosity $(\lambda>1)$, which in the present case corresponds to $\eta>0.1 \mathrm{~Pa} \cdot \mathrm{s}$. For the oils less viscous than the continuous phase $(\lambda<1)$, the calculated viscosities from drop deformation notably disagree with the measurements in the rheometer. The disagreement between both sets of data in this condition comes from the inaccuracy of Eq. 3 for small $\lambda$ (low drop viscosity), already noticed in the
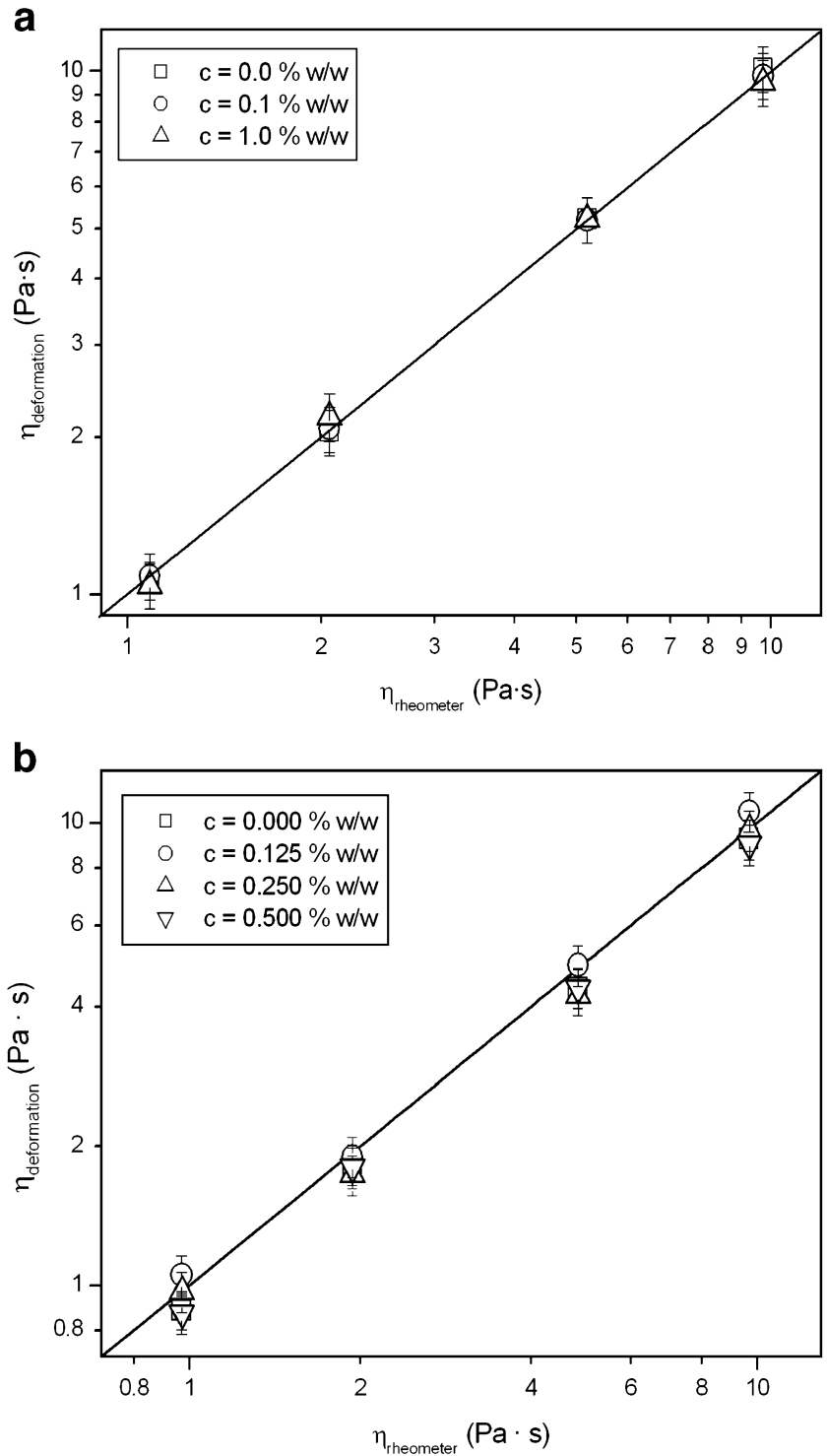

Fig. 5 Comparison of the viscosities measured in a conventional rheometer and using the drop deformation technique. a PVP solution with the different concentrations of Tween 20. b PWE solution with the different concentrations of $\mathrm{SOP} / 03$

previous application of this theory when determining, i.e., interfacial tensions (Megias-Alguacil et al. 2004a). On the other hand, the model of Taylor is robust and reliable in a wide range of experimental conditions, and, according to our previous and present studies, even when a surfactant is adsorpted at the liquid-liquid interface. The restrictive condition that guarantees the suitability of the Taylor model is that dynamical equilibrium states of deformation are fully reached by the sheared droplets, as in the present study.

\section{Conclusions}

We have explored an interesting and useful application of the drop deformation technique. Experimentally, it is shown 
than coupling two dynamical properties followed by a small drop made of a unknown liquid submitted to a simple shear flow developed by another known fluid, it is possible to extract information of the bulk properties of such a material from the behaviour of the liquid-liquid interface. These dynamical properties are, respectively, the droplet shape when a steady state is reached and its recovery process after the cessation of the flow.

In this case, the technique provides a rheometric tool which makes possible the determination of the viscosity of extremely small amounts of a Newtonian fluid, then allowing its knowledge when the conventional methods are hardly applicable due to, i.e., lack of material. This method offers a good quantitative agreement with bulk viscosity measurements in a conventional rheometer in the range $\eta_{\mathrm{d}}>\eta_{\mathrm{m}}(\lambda>1)$.

We have successfully extended the applicability of this technique to more real systems which may contain a surface-active substance (intentionally added or present as impurities or contaminants), although this method was initially proposed for surfactant-free conditions. The criteria to be rigorously observed are the following "equilibrium conditions": equilibrium adsorption of surfactant on the drop surface and reliable steady states of deformation under shear. The limits of applicability of the technique for the presence of surfactants are Newtonian fluids and small deformations (inside the Taylor linear region).

Experimental studies covering a wide range of viscosity ratios with two different surfactants at several concentrations show the suitability of the deformation technique in calculating the bulk drop viscosity even when the liquidliquid interface is covered by this kind of substance, not restricted to the nature of the systems, but experimental conditions.

\section{References}

Birkhofer B, Eischen JC, Megias-Alguacil D, Fischer P, Windhab EJ (2005) Computer controlled flow cell for the study of particle drop and drop dynamics in uniaxial linear shear flow. Ind Eng Chem Res 44:6999-7009

Eggleton CD, Pawar YP, Stebe KJ (1999) Insoluble surfactants on a drop in an extensional flor: a generalization of the stagnated surface limit to deforming interfaces. J Fluid Mech 385:79-99

Grace HP (1982) Dispersión phenomena in high viscosity inmiscible fluid systems and application of static mixers as dispersion devices in such systems. Chem Eng Commun 14:225-277

Guido S, Villone M (1999) Measurement of interfacial tension by drop retraction analysis. J Colloid Interface Sci 209:247-250

$\mathrm{Hu}$ YT, Lips A (2001) Determination of viscosity from drop deformation. J Rheol 45:1453-1463

Megias-Alguacil D, Fischer P, Windhab EJ (2004) Experimental determination of interfacial tension by different dynamical under simple shear flow conditions with a novel computer-controlled parallel bands apparatus. J Colloid Interface Sci 274:631-636

Megias-Alguacil D, Fischer P, Windhab EJ (2004) Viscosity calculated from deformation of droplets under linear shear flow. XIVth International Congress on Rheology, Seoul, Korea, 22-27 August 2004

Megias-Alguacil D, Fischer P, Windhab EJ (2006) Determination of the interfacial tension of low density difference liquid-liquid systems containing surfactants by droplet deformation methods. Chem Eng Sci 61:1386-1394

Pawar Y, Stebe KJ (1996) Marangoni effects on drop deformation in an extensional flow: the role of surfactant physical chemistry. I. Insoluble surfactants. Phys Fluids 8:1738-1751

Rallison JM (1984) The deformation of small viscous drops and bubbles in shear flows. Annu Rev Fluid Mech 16:45-66

Rumscheidt FD, Mason SG (1961) Deformation and burst of fluid drops in shear and hyperbolic flow. J Colloid Interface Sci 16:238-261

Taylor GI (1934) The formation of emulsions in definable fields of flow. Proc R Soc London Ser A 146:501-523

Wolf B, Frith WJ, Singleton S, Tassieri M, Norton IT (2001) Shear behaviour of biopolymer suspensions with spheroidal and cylindrical particles. Rheol Acta 41:238-247 